\title{
Sugar sweetened beverage consumption is positively associated with Klotho levels at two years of age in LatinX youth
}

\author{
Sofia Villagomez ${ }^{1}$, Dena B. Dubal², Jessica Hawkins ${ }^{1}$, Dan Wang ${ }^{2}$ and Janet M. Wojcicki ${ }^{1 *}$ (D
}

\begin{abstract}
Background: Klotho is an anti-aging protein mainly expressed in the kidneys with a smaller amount expressed in adipose tissue. Klotho effects include roles in reducing oxidative stress, insulin signaling, adipogenesis and glucose metabolism. Few studies have investigated the role of dietary factors such as sugar sweetened beverages (SSBs) on serum a-klotho levels in young children.

Methods: Data was collected from 60 low-income Latina pregnant women and their infants in San Francisco from birth until 2 years of life and examined for associations between dietary factors and child secreted a-klotho protein levels at 2 years.

Results: Mean a-klotho levels were $1782.96 \pm 874.56 \mathrm{pg} / \mathrm{mL}$ at 2 years of age. Any consumption of SSBs was independently associated with increased a-klotho levels (Beta $=682.79,95 \% \mathrm{Cl} 67.50,1298.09 ; p=0.03$ ). Household income ranging from $\$ 25,000$ to $\$ 50,000$ was also correlated to higher levels of a-klotho in children compared with lower income levels $(<\$ 25,000)$ (Beta $=1613.35,95 \% \mathrm{Cl} 527.37,2699.33 ; p=0.005)$.

Conclusions: The positive association between SSB intake and a-klotho levels at 2 years may reflect higher phosphate levels consistent with SSB intake. Higher socioeconomic status may be a proxy for reduced stress exposure in children, also associated with higher a-klotho levels. Future studies should evaluate the early impact of exposures to SSBs, stress and accelerated aging in children.
\end{abstract}

Keywords: Klotho, Sugar sweetened beverages, LatinX youth

\section{Background}

Klotho is a protein existing in multiple forms (membrane bound, intracellular, and secreted or $\alpha$-klotho, $\beta$ klotho, and $\gamma$-klotho), encoded by the Klotho gene [1]. $\alpha$-Klotho is the form of the protein that plays a major role in ion homeostasis and interacts with fibroblast growth factor - 23 (FGF-23), a phosphaturic hormone derived from bone, that inhibits inorganic phosphate renal uptake [1].

\footnotetext{
*Correspondence: janet.wojcicki@ucsf.edu

'Department of Pediatrics, University of California, 550 16th Street, San Francisco, USA

Full list of author information is available at the end of the article
}

The effects of $\alpha$-klotho include roles in reducing oxidative stress, insulin signaling, adipogenesis, glucose and calcium metabolism [2]. Due to its multiple roles in homeostasis, metabolism and potentially chronic disease risk, certain $\alpha$-klotho allele combinations are referred to as "anti-aging" genotypes. In mouse models, higher $\alpha$ klotho expression is associated with a longer life [1] while in humans secreted $\alpha$-klotho protein levels increased post exercise regimens [3] leading to the interpretation that it is potentially also a fitness marker in addition to a health marker. Considering the potential life-long benefits of maintaining, if not increasing secreted $\alpha$-klotho in preventing disease and supporting

C C The Author(s). 2021 Open Access This article is licensed under a Creative Commons Attribution 4.0 International License, which permits use, sharing, adaptation, distribution and reproduction in any medium or format, as long as you give appropriate credit to the original author(s) and the source, provide a link to the Creative Commons licence, and indicate if changes were made. The images or other third party material in this article are included in the article's Creative Commons licence, unless indicated otherwise in a credit line to the material. If material is not included in the article's Creative Commons licence and your intended use is not permitted by statutory regulation or exceeds the permitted use, you will need to obtain permission directly from the copyright holder. To view a copy of this licence, visit http://creativecommons.org/licenses/by/4.0/ The Creative Commons Public Domain Dedication waiver (http://creativecommons.org/publicdomain/zero/1.0/) applies to the data made available in this article, unless otherwise stated in a credit line to the data. 
metabolism it is important to understand the dietary, socioeconomic, and hormonal factors which could impact circulating levels of this important protein.

One of $\alpha$-klotho's key roles in the body is in mineral metabolism, especially phosphate and calcium [1]. Secreted $\alpha$-klotho maintains ion homeostasis by inhibiting renal and intestinal phosphate $\left(\mathrm{P}_{\mathrm{i}}\right)$ transporters to decrease plasma phosphate concentrations [1]. $\alpha$-Klotho and FGF-23 work in combination to increase urinary phosphorous and calcium reabsorption for optimal concentration of $\mathrm{CA}^{2+}$ and $\mathrm{P}_{\mathrm{i}}$ in bone and blood [4]. In normal physiology, an increase in phosphate leads to an increase of both $\alpha$-klotho and FGF-23, which suppresses IGF-1 signaling [5], an important growth hormone.

High levels of dietary phosphate have adverse consequences especially for those with impaired kidney function, but new understandings of hormonal mechanisms including FGF-23 and $\alpha$-klotho [6] promotes a mechanism of potential harm [7] without compromised kidney function. It is possible that early exposures in life to higher phosphate levels may be more detrimental than later ones. A study in mice found that during early life, a diet high in phosphate was associated with a stronger adverse effect on $\alpha$-klotho expression in the kidneys, than in mice fed the same diet during a later (peri-adolescent) growth period [7]. This is of concern for health in all populations due to the increased use of phosphate as a food additive, (e.g. processed meats, and sodas) which is now the most common dietary source of phosphate [8]. In the USA hyperphosphatemia (defined as an abnormally high serum phosphate concentration of $>4.4 \mathrm{mg} / \mathrm{dL}$ ) [9] has been found to be twice as common among persons of low income than among persons of high income likely due to the high concentration of phosphate in low-cost food items [8].

The cohort described here represents a predominantly low-income population with potentially high sugarsweetened beverage (SSB) intake. In this context, we assessed potential connections between diet and $\alpha$ klotho levels in the first few years of life.

\section{Methods}

In our previously described Latinx, Eating and Diabetes cohort (LEAD) $n=97$ of pregnant, self-identified Latina women, cord blood was collected from a subset of women $(n=72)$ as were infant anthropometrics as previously described $[10,11]$. At 2 years of age, during the time that many children have an additional blood draw to check for lead levels as part of the Special Supplemental Nutrition Program for Women, Infants and Children (WIC) program in California, children had an additional venous blood draw. Additional specifics of the study are described below.

\section{Socio-demographic and clinical data}

Maternal socio-demographics including race, ethnicity, age, marital status and employment were collected at enrollment as previously described $[10,11]$. Women were assessed for prenatal depression using the Edinburgh Postnatal Depression Scale (EPDS) and the Center for Epidemiological Depression Scale (CESD) in pregnancy [11]. Infant anthropometrics collected at birth using standard digital scales and tape measures and birth characteristics were extracted from the medical record (Apgar scores and gestational age) [11]. Infant anthropometrics (weight and length/height) were similarly assessed at 6 months, 12 months, and 2 years of age. Standard Center for Disease Control (CDC) growth curves were used to define childhood obesity as weight for length or body mass index (BMI) percentile $\geq 95$ th $[11,12]$. Food frequency questionnaires assessed infant and toddler dietary intake at 4-6 weeks, 6 months, 1 and 2 years of age. Exclusive breastfeeding was defined as consumption of only breastmilk without the addition of any other foods or beverages including water using the World Health Organization (WHO) definition [13]. SSBs were defined as any beverage with added sugars included sodas, fruit drinks, sweetened milk beverages and sports drinks. High intake of $100 \%$ fruit juice consumption was defined as more than 4 servings per week and high intake of SSBs was defined as more than 4 servings per week. All women provided written consent for their own and their children's participation as all children were below the age of written and verbal consent. The study was approved by the Institutional Review Board (IRB) of the University of California, San Francisco (Committee on Human Research (CHR)).

\section{Laboratory}

Briefly, serum leptin, insulin, insulin-like growth factor 1 (IGF-1) were assayed (Milliplex MAP Human Metabolic Hormone Magnetic Bead Panel [Millipore], Linco, St Charles, MO, USA). The minimum detectable concentration for leptin was $27 \mathrm{pg} / \mathrm{mL}$ and insulin $58 \mathrm{pg} / \mathrm{mL}$. The intra-assay coefficient of variation $(\mathrm{CV})$ and interassay CV for leptin and insulin were 3 and $4 \%$ respectively. Serum IGF-1 was assessed using the Milliplex MAP Human IGF-1 Single Plex. The minimum detectable concentration was $52 \mathrm{pg} / \mathrm{mL}$ and the intra-assay CV was $4 \%$ and the inter-assay was $7 \%$.

Serum $\alpha$-klotho levels were measured in plasma using a solid-phase sandwich enzyme-linked immunosorbent assay (Immuno-Biological Laboratories, Takasaki, Japan) by the Dubal lab at UCSF as previously described [11]. All samples were run in duplicate. The assay had an average intra-assay $\mathrm{CV}$ of approximately $3-5 \%$. Samples with a $\mathrm{CV}$ above $7 \%$ were repeated with 16 samples 
being re-run and the control sample in the second run being in close agreement with the first run.

\section{Statistical methods}

$\alpha$-Klotho values at 2 years of age, as the primary outcome of interest, were assessed for normal distribution through graphing and tests for normality including Shapiro-Wilk and Shapiro-Francia tests [11]. As the data was not normally distributed, we used nonparametric tests of association. Wilcoxon rank sum (Mann-Whitney) test was used for dichotomous predictors of $\alpha$-klotho levels and Kruskal-Wallis for categorical predictors [11]. Association between $\alpha$-klotho levels and continuous predictors were assessed with Spearman's rank correlation coefficients $(\rho)$. Statistical significance was determined at $P$-values $<0.05$. Data in tables are expressed as means plus or minus standard deviations and percentages [11]. We also assessed normality for linear regression models. Linear regression residuals were not normally distributed. We tested a bootstrapped sample with 1000 replications and compared findings to regression coefficients which were not bootstrapped. As there were no differences in regression results between the bootstrapped and non-bootstrapped finding, we assumed that departures from normality were related to sample size and not to other factors. As such, we used linear regressions to test associations.

Multivariable analyses included those variables that were significant at $p<0.20$, a threshold chosen to reduce the possibility of Type II errors, in addition to those variables that previously demonstrated a plausible association with $\alpha$-klotho levels including insulin and leptin levels. We did not include variables that had a small cell size $<5$ in bivariate analysis in our multivariable models. Correlation tests were run on all variables and any which showed $\mathrm{R}>0.7$ were not included simultaneously in models. Beta coefficients are presented with 95\% confidence intervals $(\mathrm{CI})$ and $p$-values. Robust multivariable regression models were subsequently compared with initial models to assess for the possible influence of outliers.

\section{Results}

Of the 97 parent/child dyads included in the LEAD cohort, secreted $\alpha$-klotho protein measurements taken at 2 years were available for 60 (62\% of the sample) children. Mean $\alpha$-klotho levels were $1783.0 \pm 874.6 \mathrm{pg} / \mathrm{mL}$ at 2 years of age. There were few differences between families who had child $\alpha$-klotho measured at two years of age and those who did not including maternal age $(p=$ $0.09)$, child birthweight percentile $(p=0.75)$, family income $(p=0.93)$, and maternal marital status $(p=0.44)$. Families who did have $\alpha$-klotho measured were more likely to be Mexican versus Central or South American origin $(p<0.01)$ and have higher maternal education levels $(p=0.03)$.

\section{Maternal characteristics}

Increasing maternal education level was associated with higher $\alpha$-klotho levels with children of high school graduates having $2359.83 \pm 1387.12 \mathrm{pg} / \mathrm{mL}$ compared to $1690.28 \pm 755.59 \mathrm{pg} / \mathrm{mL}(p=0.045)$ for the children of mothers with less than a high school education (Table 1). There was no association between other maternal demographic characteristics and $\alpha$-klotho levels including maternal age or health during/prior to pregnancy including pre-pregnancy BMI, or weight gain in pregnancy (Table 1). Self-reported family income levels $25 \mathrm{~K}-50 \mathrm{~K}$ at time of enrollment neared statistical significance for higher child levels of $\alpha$-klotho compared to family income of $<$ $25 \mathrm{~K}(2377.70 \pm 1758.81 \mathrm{pg} / \mathrm{mL}$ versus $1716.88 \pm 717.72$ $\mathrm{pg} / \mathrm{mL})(p=0.079$; Table 1$)$.

\section{Child characteristics}

We did not find any association between birth characteristics including child sex, gestational age, Apgar score at 5 min and $\alpha$-klotho levels. However, birthweight percentile below the 50th percentile neared statistical significance for increased $\alpha$-klotho levels compared with birthweight percentile greater or equal to the 50th percentile $(2133.42 \pm 1079.41 \mathrm{pg} / \mathrm{mL}$ versus $1448.03 \pm 283.36 \mathrm{pg} / \mathrm{mL}$ $p=0.055$; Table 2). Other birth anthropometrics including being macrosomic $(>4000 \mathrm{~g})$, underweight $(<2500 \mathrm{~g})$ or preterm $(<37$ weeks gestational age $)$ were not associated with $\alpha$-klotho levels at 2 years of age. Similarly, head circumference percentile and obesity in early childhood ( 6 months, 12 months, 24 months) were not associated with $\alpha$-klotho levels at 2 years of age. We also did not find any association between infant and toddler adiposity or growth hormone levels taken at birth or two years (IGF-1, ghrelin, insulin, leptin) and $\alpha$-klotho levels at 2 years. Male children tended to have higher $\alpha$-klotho levels (Beta $=-$ 396.21, 95\%CI -1064.77-272.35; $p=0.24$ ) but the association was not statistically significant (Table 2).

\section{Child dietary factors}

There was also no association between breastfeeding (exclusive or any), any formula intake, 100\% juice intake and $\alpha$-klotho levels at 2 years of age. Exclusive breastfeeding trended towards statistical significance with higher $\alpha$-klotho levels among those infants being exclusively breastfed at six months (defined by WHO as: "no other food or drink, not even water, except breast milk for 6 months of life, but allows the infant to receive ORS, drops and syrups [13]") versus those that were not $(2561.18 \pm 1384.99 \mathrm{pg} / \mathrm{mL}$ versus $1754.47 \pm 851.26 \mathrm{pg} /$ $\mathrm{mL} ; p=0.126$; Table 3). At 24 months of age, any SSB consumption was associated with higher $\alpha$-klotho levels 
Table 1 Maternal Characteristics and a-Klotho Levels at 2 Years

\begin{tabular}{|c|c|c|c|}
\hline Variable & $\begin{array}{l}\text { Population mean } \pm \text { Standard Deviation } \\
\text { or } \mathrm{n} \text { /total }\end{array}$ & $\begin{array}{l}\text { a-Klotho levels at } 2 \text { years mean } \pm \text { standard } \\
\text { deviation, } \mathrm{pg} / \mathrm{mL}\end{array}$ & $\begin{array}{l}p \text { - } \\
\text { value }\end{array}$ \\
\hline \multicolumn{4}{|l|}{ Health Characteristics } \\
\hline $\begin{array}{l}\text { Pre-Pregnancy body mass index } \\
(\mathrm{BMI}), \mathrm{kg} / \mathrm{m} 2\end{array}$ & $27.52 \pm 4.62$ & $1789.42 \pm 898.99$ & 0.83 \\
\hline Underweight or Normal & $12 / 55(22)$ & $1852.09 \pm 1214.14$ & \\
\hline Overweight & $22 / 55(40)$ & $1682.67 \pm 710.73$ & \\
\hline Obese & 21/55 (38) & $1865.44 \pm 905.52$ & \\
\hline Excess weight gain in pregnancy & & & 0.18 \\
\hline No & $27 / 54(50)$ & $1591.09 \pm 465.31$ & \\
\hline Yes & $27 / 54(50)$ & $1906.52 \pm 1109.41$ & \\
\hline Depressive symptoms in pregnancy & & & 0.79 \\
\hline No & $48 / 60(80)$ & $1798.40 \pm 894.30$ & \\
\hline Yes & $12 / 60(20)$ & $1721.19 \pm 824.66$ & \\
\hline Age of mom at time of delivery & $28.41 \pm 5.34$ & $1782.96 \pm 874.56$ & 0.74 \\
\hline$<35$ years & $50 / 60(83)$ & $1819.18 \pm 919.34$ & \\
\hline$>=35$ years & $10 / 60(17)$ & $1601.85 \pm 607.32$ & \\
\hline \multicolumn{4}{|l|}{ Socio-Demographics } \\
\hline \multicolumn{4}{|l|}{ Maternal Education } \\
\hline Less than high school & $51 / 59(86)$ & $1690.28 \pm 755.59$ & \\
\hline High school graduate or more & 8/59 (14) & $2359.83 \pm 1387.12$ & 0.05 \\
\hline \multicolumn{4}{|l|}{ Marital Status } \\
\hline $\begin{array}{l}\text { Single or divorced/eparated/ } \\
\text { widowed }\end{array}$ & $9 / 60(15)$ & $1565.23 \pm 876.99$ & \\
\hline Living with partner or married & $51 / 60(85)$ & $1821.37 \pm 877.18$ & 0.42 \\
\hline \multicolumn{4}{|l|}{ Self-Reported Household Income } \\
\hline Less than 25,000 & $54 / 60(90)$ & $1716.88 \pm 717.72$ & \\
\hline $25,000-50,000$ & $6 / 60(10)$ & $2377.70 \pm 1758.81$ & 0.08 \\
\hline
\end{tabular}

$(2029.78 \pm 999.45$ versus $1402.78 \pm 496.28 ; \quad p<0.01$; Table 3).

\section{Multivariable analysis}

Adjusting for weight z-score at birth, leptin levels at 2 years, insulin levels at birth, maternal education level, household income, and child sex, any consumption of SSBs at 24 months was associated with increased $\alpha$ klotho levels in children (Beta $=682.79$, 95\%CI 67.50, 1298.09.1; $p=0.03$ ) (Table 4). Household income ranging from $\$ 25,000$ to $\$ 50,000$ per year was also significantly associated with higher levels of childhood $\alpha$ klotho levels compared to those with lower income levels (Beta $=1613.35$, 95\%CI 527.37, 2699.33; $p=0.005$ ) (Table 4). When insulin was excluded from our final multivariable regression to provide more power and a larger sample size, SSB intake approached significance $($ Beta $=434.68,95 \%$ CI $-20.33,889.68 ; p=0.06)$ as did higher maternal education level (Beta $=647.57$; 95\% CI -53.41-1348.55; $p=0.07$ ) and higher household income
$($ Beta $=696.78 ; \quad 95 \%$ CI $\quad-69.28-1462.85 \quad p=0.074)$ (Table 5).

\section{Discussion}

Stress, income and Klotho

We found a significant positive association between $\alpha$ klotho levels at 2 years and increased household income possibly related to increased stress exposures in lower income households. In a previous study examining psychosocial factors in adults in relation to $\alpha$-klotho levels, high-stress caregivers had lower serum $\alpha$-klotho levels than low-stress ones [14]. Lower $\alpha$-klotho levels were also significantly associated with depressive symptoms, a common maladaptive brain response to stress [14]. Other studies have shown that poverty increases stress responses not only in parents [15] but children [15] as well, affecting both physiological [16] and psychological [17] development even at very young ages. Efforts to alleviate poverty have been suggested to improve health outcomes in children [16]. In our LEAD cohort, mothers 
Table 2 Child Characteristics and a-Klotho Levels at 2 Years of Age

\begin{tabular}{|c|c|c|c|}
\hline Variable & $\begin{array}{l}\text { Population mean } \pm \text { Standard Deviation or } \\
\mathrm{n} / \text { total }\end{array}$ & $\begin{array}{l}\text { a-Klotho levels at } 2 \text { years mean } \pm \text { standard } \\
\text { deviation, } \mathrm{pg} / \mathrm{mL}\end{array}$ & $\begin{array}{l}p- \\
\text { value }\end{array}$ \\
\hline \multicolumn{4}{|l|}{ Birth Characteristics } \\
\hline \multicolumn{4}{|l|}{ Child sex } \\
\hline Female & $30 / 60(50)$ & $1900.78 \pm 860.19$ & \multirow[t]{2}{*}{0.30} \\
\hline Male & $30 / 60(50)$ & $1665.14 \pm 887.38$ & \\
\hline Gestational age (weeks) & $39.12 \pm 1.37$ & $1782.96 \pm 874.56$ & \multirow[t]{3}{*}{0.25} \\
\hline$<40$ & $44 / 60(73)$ & $1735.59 \pm 828.01$ & \\
\hline$>=40$ & $16 / 60(27)$ & $1913.22 \pm 1009.15$ & \\
\hline Gestational age (tertiles) & $39.12 \pm 1.37$ & $1782.96 \pm 874.56$ & \multirow[t]{4}{*}{0.25} \\
\hline$<39$ & 23/60 (39) & $1653.50 \pm 925.44$ & \\
\hline $39-39.8$ & 20/60 (33) & $1852.85 \pm 725.43$ & \\
\hline$>39.8$ & $17 / 60(28)$ & $1875.75 \pm 989.24$ & \\
\hline Apgar score at 5 min & $8.8 \pm 0.6$ & $1782.96 \pm 874.56$ & \multirow[t]{3}{*}{0.55} \\
\hline$<9$ & $11 / 60(18)$ & $1527.74 \pm 448.58$ & \\
\hline$>=9$ & $49 / 60(82)$ & $1840.25 \pm-938.05$ & \\
\hline \multicolumn{4}{|l|}{ Birth Anthropometrics } \\
\hline Birth weight, g & $3384.09 \pm 471.41$ & $1782.96 \pm 874.56$ & 0.41 \\
\hline \multicolumn{4}{|l|}{ Macrosomic at birth (> $4000 \mathrm{~g}$ ) } \\
\hline No & $57 / 60(95)$ & $1779.65 \pm 891.88$ & \multirow[t]{2}{*}{0.90} \\
\hline Yes & $3 / 60(5)$ & $1845.77 \pm 533.61$ & \\
\hline \multicolumn{4}{|c|}{ Underweight ( $<2500 \mathrm{~g})$ or preterm ( $<37$ weeks) } \\
\hline No & $54 / 60(90)$ & $1820.17 \pm 910.91$ & \multirow[t]{2}{*}{0.33} \\
\hline Yes & $6 / 60(10)$ & $1448.03 \pm 283.36$ & \\
\hline Birth weight percentile & $56.53+/-25.40$ & $1782.96 \pm 874.56$ & 0.05 \\
\hline$<50 \%$ & $24 / 60(40)$ & $2133.42 \pm 1079.41$ & \multirow[t]{2}{*}{0.01} \\
\hline$>=50 \%$ & $36 / 60(60)$ & $1549.31 \pm 619.12$ & \\
\hline \multicolumn{4}{|c|}{ Birth weight for length below 5 th $\%$ ile } \\
\hline No & $51 / 60(85)$ & $1846.64 \pm 919.01$ & \multirow[t]{2}{*}{0.18} \\
\hline Yes & $9 / 60(15)$ & $1422.09 \pm 435.82$ & \\
\hline $\begin{array}{l}\text { Head circumference percentile } \\
\text { (tertiles) }\end{array}$ & $34.20+/-1.42$ & $1782.96 \pm 874.56$ & 0.38 \\
\hline$<25 \%$ & $36 / 60(60)$ & $1511.39 \pm 543.04$ & \\
\hline $25-50 \%$ & $18 / 60(30)$ & $2398.09 \pm 1199.40$ & \\
\hline$>50 \%$ & $6 / 60(10)$ & $1566.93 \pm 347.65$ & \\
\hline
\end{tabular}

\section{Early childhood anthropometrics}

Obesity in early childhood

$\begin{array}{ll}6 \text { months (Yes) } & 7 / 60(12) \\ \text { No } & 53 / 60(88) \\ 12 \text { months (Yes) } & 7 / 60(12) \\ \text { No } & 53 / 60(88) \\ 24 \text { months (Yes) } & 6 / 60(10) \\ \text { No } & 52 / 60(90)\end{array}$

$1681.31 \pm 685.04$

$1796.38 \pm 901.17$

$1656.52 \pm 761.34$

$1799.65 \pm 893.59$

$1975.48 \pm 790.31$

Hormone Levels
IGF-1 at birth $(\mathrm{pg} / \mathrm{mL})$
$30,749.25 \pm 21,214.53$
$<17,600$
$14 / 46(31)$

$1756.77 \pm 888.70$

$1792.06 .39 \pm 953.16$ 
Table 2 Child Characteristics and a-Klotho Levels at 2 Years of Age (Continued)

\begin{tabular}{|c|c|c|c|}
\hline Variable & $\begin{array}{l}\text { Population mean } \pm \text { Standard Deviation or } \\
\mathrm{n} / \text { total }\end{array}$ & $\begin{array}{l}\text { a-Klotho levels at } 2 \text { years mean } \pm \text { standard } \\
\text { deviation, } \mathrm{pg} / \mathrm{mL}\end{array}$ & $\begin{array}{l}p \text { - } \\
\text { value }\end{array}$ \\
\hline $17,600-35,120$ & $17 / 46(40)$ & $1814.85 \pm 901.79$ & \\
\hline$>35,120$ & 15/46 (33) & $1983.53 \pm 1209.86$ & \\
\hline IGF-1 at 2 years & $29,126.19 \pm 15,992.23$ & $1782.96 \pm 874.56$ & 0.42 \\
\hline$<17,600$ & $18 / 60(30)$ & $1597.60 \pm 663.41$ & 0.54 \\
\hline $17,600-35,120$ & $27 / 60(45)$ & $1893.88 \pm 1020.88$ & \\
\hline$>35,120$ & $15 / 60(25)$ & $1805.73 \pm 827.61$ & \\
\hline Ghrelin at birth (pg/mL) & $39 \pm 22$ & $1499.55 \pm 367.95$ & 0.40 \\
\hline$<22$ & 4/13 (31) & $1544.74 \pm 563.03$ & 0.85 \\
\hline $22-45$ & $7 / 13(54)$ & $1444.72 \pm 268.62$ & \\
\hline$>45$ & 2/13 (15) & $1601.06 \pm 426.68$ & \\
\hline Insulin at birth $(\mathrm{pg} / \mathrm{mL})$ & $953.76 \pm 546.78$ & $1801.79 \pm 985.83$ & 0.18 \\
\hline$>750$ & $17 / 41(41)$ & $1823.06 \pm 1112.10$ & 0.65 \\
\hline $750-1060$ & 16/41 (39) & $1920.01 \pm 986.86$ & \\
\hline$>1060$ & $8 / 41(20)$ & $1520.18 \pm 718.30$ & \\
\hline Insulin at 2 years $(\mathrm{pg} / \mathrm{mL})$ & $766.17 \pm 900.33$ & $1782.96 \pm 874.56$ & 0.35 \\
\hline$>750$ & $41 / 60(68)$ & $1706.81 \pm 888.29$ & 0.60 \\
\hline $750-1060$ & 8/60 (13) & $1895.41 \pm 736.32$ & \\
\hline$>1060$ & $11 / 60(18)$ & $1984.96 \pm 947.67$ & \\
\hline Leptin at birth $(\mathrm{pg} / \mathrm{mL})$ & $23,025.87 \pm 18,375.04$ & $1766.25 \pm 989.08$ & 0.82 \\
\hline$<11,200$ & $12 / 39(30)$ & $1827.42 \pm 984.01$ & 0.70 \\
\hline $11,200-27,000$ & 17/39 (44) & $1618.32 \pm 1063.94$ & \\
\hline$>27,000$ & 10/39 (26) & $1944.32 \pm 923.65$ & \\
\hline Leptin at 2 years $(\mathrm{pg} / \mathrm{mL})$ & $733.93 \pm 1267.03$ & $1782.96 \pm 874.56$ & 0.85 \\
\hline$<3000$ & $58 / 60$ (96) & $1789.65 \pm 884.72$ & 0.72 \\
\hline $3000-6000$ & $1 / 60(2)$ & 1123.12 & \\
\hline$>8000$ & $1 / 60(2)$ & 2054.59 & \\
\hline
\end{tabular}

who had higher housheold incomes may have had children who experienced less chronic stress and therefore had higher $\alpha$-klotho levels, than children in lower income households and higher exposures to stress.

\section{Sugar sweetened beverages}

We found a positive association between SSB intake and higher $\alpha$-klotho levels supporting a potential association between secreted $\alpha$-klotho levels and phosphate in young, low-income children $[18,19]$. Children at 24 months of age who reported drinking any SSBs showed significantly higher $\alpha$-klotho levels $(2029.78 \pm 999.45 \mathrm{pg} / \mathrm{mL})$ than those who did not $(1402.78 \pm 496.28 \mathrm{pg} / \mathrm{mL})$ even when controlling for weight $\mathrm{Z}$ score at birth, leptin levels at two years, insulin at birth, child sex, maternal education and household income levels. Our findings suggest that dietary sources of phosphate as a food additive can influence this relationship [8]. Phosphate is commonly used as an additive in sugary drinks marketed to children and especially colas [8], commonly consumed by young children, particularly in higher risk low-income communities. Fifty-nine percent of our cohort was already consuming SSBs by 2 years of age.

Phosphorous additives on food labels can be difficult to decipher and, the amount of phosphorous in sugar sweetened beverages can range from $<1 \mathrm{mg}$ to $175 \mathrm{mg}$ per serving [20]. Even if parents in our study were avoiding giving their children carbonated sodas, the drinks they may have assumed were better alternatives contain high amounts of phosphorous per serving: Hawaiian Punch (175 mg), Fruit Works (63 mg-140), Aquafina Flavor (62 mg-85 mg), Tropicana Fruit Punch (93 mg) [20]. Even amounts of approximately $60 \mathrm{mg}$ per serving serve as significant sources of dietary phosphates. The organic absorption rate of phosphates from meats, dairy, grains and beans is $40-60 \%$ [21] while the absorption rate from inorganic phosphates in SSBs, processed meats and cheeses [15] can be as high as 100\% [22]. 
Table 3 Child Dietary Intake and a-Klotho Levels at 2 Years

\begin{tabular}{|c|c|c|c|}
\hline Variable & Population mean +/- Standard Deviation or n/total & $\begin{array}{l}\text { a-Klotho levels at } 2 \text { years mean }+/- \text { standard deviation, } \\
\mathrm{pg} / \mathrm{mL}\end{array}$ & $p$-value \\
\hline \multicolumn{4}{|l|}{ Breastfeeding } \\
\hline \multicolumn{4}{|l|}{ Any } \\
\hline 4-6 weeks (Yes) & $59 / 60(98)$ & $1795.15 \pm 876.92$ & 0.70 \\
\hline No & $1 / 60(2)$ & 1063.83 & \\
\hline 6 months (Yes) & $42 / 58(72)$ & $1825.55 \pm 900.11$ & 0.69 \\
\hline No & $16 / 58(28)$ & $1719.15 \pm 874.44$ & \\
\hline 12 months (Yes) & $36 / 59(61)$ & $1759.55 \pm 756.31$ & 0.70 \\
\hline No & 23/59 (39) & $1850.87 \pm 1054.50$ & \\
\hline \multicolumn{4}{|l|}{ Exclusive } \\
\hline 6 months (Yes) & $3 / 58(5)$ & $2561.18 \pm 1384.99$ & 0.13 \\
\hline No & $55 / 58(95)$ & $1754.47 \pm 851.26$ & \\
\hline 12 months (Yes) & 20/60 (33) & $1875.99 \pm 850.63$ & 0.56 \\
\hline No & $40 / 60(66)$ & $1736.44 \pm 893.27$ & \\
\hline \multicolumn{4}{|l|}{ Infant Formula } \\
\hline 4-6 weeks (Yes) & $31 / 60(52)$ & $1717.74 \pm 933.12$ & 0.55 \\
\hline No & 29/60 (48) & $1852.66 \pm 817.94$ & \\
\hline \multicolumn{4}{|l|}{ 100\% Fruit Juice } \\
\hline \multicolumn{4}{|l|}{ Any } \\
\hline 6 months (yes) & $17 / 57(30)$ & $1608.98 \pm 694.53$ & 0.30 \\
\hline No & $40 / 57(70)$ & $1878.06 \pm 963.75$ & \\
\hline 12 months (yes) & 46/59 (78) & $1769.26 \pm 883.68$ & 0.67 \\
\hline No & $13 / 59(22)$ & $1886.74 \pm 881.32$ & \\
\hline 24 months (yes) & 55/56 (98) & $1803.69 \pm 890.42$ & 0.28 \\
\hline No & $1 / 56(2)$ & 832.76 & \\
\hline \multicolumn{4}{|l|}{ High Intake } \\
\hline 24 months (yes) & $18 / 56(32)$ & $1681.84 \pm 669.55$ & 0.55 \\
\hline No & $38 / 56(68)$ & $1835.85 \pm 984.04$ & \\
\hline \multicolumn{4}{|c|}{ Sugar Sweetened Beverages } \\
\hline \multicolumn{4}{|l|}{ Any } \\
\hline 12 months (yes) & $17 / 59(29)$ & $1631.34 \pm 584.62$ & 0.37 \\
\hline No & $42 / 59(71)$ & $1861.45 \pm 968.93$ & \\
\hline 24 months (yes) & $34 / 58(59)$ & $2029.78 \pm 999.45$ & 0.01 \\
\hline No & $24 / 58(41)$ & $1402.78 \pm 496.28$ & \\
\hline \multicolumn{4}{|l|}{ High Intake } \\
\hline 24 months (yes) & 9/58 (16) & $1603.39 \pm 859.46$ & 0.54 \\
\hline No & 49/58 (84) & $1800.99 \pm 889.21$ & \\
\hline
\end{tabular}


Table 4 Final Multivariable Model Summary

\begin{tabular}{|c|c|c|c|c|c|c|}
\hline & & & Numbe & Observation & $s=$ & 39 \\
\hline & & & & Probability & $F=$ & 0.0119 \\
\hline & & & & R-Squared $=$ & & 0.4177 \\
\hline & & & & Adjusted R-S & yuared = & 0.2862 \\
\hline & & & & Root MSE = & & 853.8 \\
\hline a-Klotho Levels at 2 years & Coefficient & Standard Error & t & $P>|t|$ & {$[95 \%$ Conf } & \\
\hline Weight Z Score at Birth & -17.15 & 219.30 & -0.08 & 0.94 & -464.41 & 430.11 \\
\hline Leptin at 2 Years & -0.04 & 0.09 & -0.41 & 0.68 & -0.23 & 0.15 \\
\hline Insulin at Birth & -0.14 & 0.35 & -0.41 & 0.68 & -0.85 & 0.56 \\
\hline SSB Intake at 2 years (yes) & 682.79 & 301.69 & 2.26 & 0.03 & 67.50 & 1298.09 \\
\hline Child Sex (male) & -396.21 & 327.80 & -1.21 & 0.24 & -1064.77 & 272.35 \\
\hline Maternal Education (high school graduate or more) & 373.68 & 449.38 & 0.83 & 0.41 & -542.83 & 1290.20 \\
\hline Household Income (25 k-50 k) & 1613.35 & 532.47 & 3.03 & 0.005 & 527.37 & 2699.33 \\
\hline
\end{tabular}

The physiological regulation of phosphate is controlled by the FGF-23/ $\alpha$-klotho mechanism such that an increase in phosphate levels is associated with an increase in both FGF-23 $[23]^{24}$ and $\alpha$-klotho levels $[1,6]$. An increase in phosphate levels signals the FGF-23 complex to trigger a cascade which reduces the number of sodium-dependent phosphate $(\mathrm{NaPi})$ cotransporters, type-2a (NaPi-2a), on the brush border membrane of proximal tubules, thereby promoting renal phosphate excretion [24]. $\alpha$-Klotho is required for FGF-23 to induce negative phosphate balance in this mechanism, demonstrated in previous studies where FGF-23 recombinant protein is unable to reduce serum phosphate levels in klotho deficient mice [24]. While $\alpha$-klotho is a necessary co-receptor of phosphate regulation [25], $\alpha$-klotho also exhibits effects independent of FGF-23 and parathyroid hormone (PTH), which regulate calcium ion homeostasis in the kidneys [23]. While previous evidence supports that high $\alpha$-klotho levels have positive physiological effects [25] and benefits to cognition [26], the elevation of $\alpha$-klotho levels via activation of the
FGF-23 phosphate regulation pathway raises concern of potential long term harm.

As significant sources of inorganic phosphates which are readily absorbed, SSBs create an artificial increase in FGF-23. Elevated FGF-23 is associated with negative health consequences including inflammation [23] and is an early marker for chronic kidney disease [4]. It is not clear whether the positive effects of increased $\alpha$-klotho levels would compensate for potential harm of chronically elevated FGF-23 levels. Our previous study found that insulin at birth was inversely correlated with cord blood $\alpha$-klotho levels [11] .There may be a confounding relationship between $\alpha$-klotho, SSBs and insulin. Controlling for insulin in our study strengthened the relationship between $\alpha$-klotho and SSBs . Future studies should also assess FGF-23 in addition to $\alpha$-klotho levels.

Any consumption of SSBs in children, may be positively associated with $\alpha$-klotho levels supporting previous studies that high phosphate diets and FGF-23/ $\alpha$-klotho mechanisms are related $[9,18]$. The initial increase in $\alpha$ klotho may be an immediate response in the short term

Table 5 Final Multivariable Model Summary, Excluding Insulin at Birth

\begin{tabular}{|c|c|c|c|c|c|c|}
\hline & & & Numb & Observations & & 57 \\
\hline & & & & Probability & $F=$ & 0.0121 \\
\hline & & & & R-Squared $=$ & & 0.2698 \\
\hline & & & & Root MSE = & & 802.94 \\
\hline a-Klotho levels at 2 years & Coefficient & Standard Error & $\mathrm{t}$ & $P>|t|$ & [95\% Cor & val] \\
\hline Weight Z Score at Birth & -143.87 & 133.16 & -1.08 & 0.28 & -411.34 & 123.59 \\
\hline Leptin at 2 Years & -0.06 & 0.08 & -0.65 & 0.52 & -0.23 & 0.12 \\
\hline SSB Intake at 2 years (yes) & 434.68 & 226.53 & 1.92 & 0.06 & -20.33 & 889.68 \\
\hline Child Sex (male) & -376.17 & 227.69 & -1.65 & 0.10 & -833.51 & 81.17 \\
\hline Maternal Education (high school graduate or more) & 647.57 & 348.99 & 1.86 & 0.07 & -53.41 & 1348.55 \\
\hline Household Income (25 k-50 k) & 696.78 & 381.40 & 1.83 & 0.07 & -69.28 & 1462.85 \\
\hline
\end{tabular}


to control phosphate levels [22] but could have lasting negative effects. High FGF-23 concentrations are linked to cardiovascular damage and inflammation [4]. Intake in early childhood could be particularly damaging as high dietary phosphates has been shown to be more detrimental at critical growth periods [7].

Dietary habits are known to be established early in life and previous evidence supports that diet quality tracks and declines from early childhood through adolesence [27]. SSB consumption in early childhood could track through adolescence [27] especially with increased exposure to advertising [28] leading to a lifetime of increased consumption [29].

\section{Limitations}

Future studies should include additional metabolic and micronutrient serum indicators. We did not assess serum phosphate, FGF-23 or vitamin D levels in our participants. These serum markers would have provided a more comprehensive understanding of the relationship between dietary phosphate intake, including inorganic phosphate from SSBs, $\alpha$-klotho and metabolic profile including Vitamin D status [1]. Further, future studies should account for all dietary sources of phosphate including those from other high phosphate foods (e.g. beans, lentils, pasta and rice) as well as intake of other source sources of inorganic phosphate from fast foods, other processed foods and canned foods.

\section{Conclusions}

The positive association between SSB intake and $\alpha$ klotho levels at 2 years may reflect higher phosphate levels consistent with SSB intake in healthy children. The early intake of SSBs may establish potentially lifelong habits of consumption. Poteintial associations between inorganic phosphates found in SSBs and $\alpha$-klotho protein may result in increased inflammation, suggesting that there may be a complex interplay between dietary intake and $\alpha$-klotho protein levels. We additionally found evidence of a possible relationship between family stress or low socioeconomic status and lower serum $\alpha$-klotho levels, similar to other published findings. The complex relationship between environmental exposures including psychosocial and dietary ones and child serum $\alpha$-klotho levels necessitates further longitduinal study at different ages.

\section{Abbreviations}

BMI: Body mass index; Cl: Confidence interval; CV: Coefficient of variation; FGF-23: Fibroblast growth factor - 23; NaPi: Sodium-dependent phosphate; SSB: Sugar sweetened beverages; PTH: Parathyroid Hormone; WIC: Special Supplemental Program for Women, Infants and Children

Acknowledgements

Not Applicable.

\section{Authors' contributions}

DBD and DW contributed to the conceptualization of the final project. SV and JMW conceptualized the paper as well as were major contributors to writing the manuscript. SV and JMW were assisted in data analysis by JH. DBD and DW generated klotho data by running the klotho assay.All authors approve the final manuscript.

\section{Funding}

NIDDK 080825; Hellman Family Foundation and Marc and Lynn Benioff. Funding played no role in the study design, data collection, analysis, and interpretation, or writing the manuscript.

\section{Availability of data and materials}

Data is publicly available by written request and after review from Janet M Wojcicki (janet.wojcicki@ucsf.edu).

\section{Declarations}

Ethics approval and consent to participate

All participants signed written, informed consent and the study was approved by the Committee on Human Research (CHR) at the University of California, San Francisco. Parents signed written, informed consent for their participation and that of their children.

\section{Consent for publication}

Not Applicable.

\section{Competing interests}

The authors declare that they have no competing interests.

\section{Author details}

${ }^{1}$ Department of Pediatrics, University of California, 550 16th Street, San Francisco, USA. ${ }^{2}$ Department of Neurology, University of California, San Francisco, USA.

Received: 13 September 2020 Accepted: 22 March 2021

Published online: 23 April 2021

\section{References}

1. Kim J-H, Hwang K-H, Park K-S, Kong ID, Cha S-K. Biological Role of Antiaging Protein Klotho. J Lifestyle Med. 2015;5(1):1-6. https://doi.org/10.15280/ jlm.2015.5.1.1.

2. Ohnishi M, Kato S, Akiyoshi J, Atfi A, Razzaque MS. Dietary and genetic evidence for enhancing glucose metabolism and reducing obesity by inhibiting klotho functions. FASEB J. 2011;25(6):2031-9. https://doi.org/10.1 096/fj.10-167056.

3. Amaro-Gahete FJ, de-la-O A, Jurado-Fasoli L, Ruiz JR, Castillo MJ, Gutiérrez Á Role of Exercise on S-Klotho Protein Regulation: A Systematic Review. Curr Aging Sci. 2019;11(2):100-7. https://doi.org/10.2174/18746098116661807021 01338.

4. Bjornstad P, Maahs DM. Diabetes complications in childhood diabetes: new biomarkers and technologies. Curr Pediatr Rep. 2015;3(2):177-86. https://doi. org/10.1007/s40124-015-0081-0.

5. Somm E, Henry H, Bruce SJ, et al. $\beta$-Klotho deficiency protects against obesity through a crosstalk between liver, microbiota, and brown adipose tissue. JCI Insight. 2017;2(8):e91809. https://doi.org/10.1172/jci.insight.91809.

6. Kuro-o M. The Klotho proteins in health and disease. Nat Rev Nephrol. 2019; 15(1):27-44. https://doi.org/10.1038/s41581-018-0078-3.

7. Fukuda-Tatano S, Yamamoto $\mathrm{H}$, Nakahashi $\mathrm{O}$, et al. Regulation of a-Klotho Expression by Dietary Phosphate During Growth Periods. Calcif Tissue Int. 2019;104(6):667-78. https://doi.org/10.1007/s00223-019-00525-0.

8. Ritz E, Hahn K, Ketteler M, Kuhlmann MK, Mann J. Phosphate Additives in Food. Dtsch Aerzteblatt Online. Published online January 27, 2012. https:// doi.org/10.3238/arztebl.2012.0049.

9. Wojcicki JM. Hyperphosphatemia is associated with anemia in adults without chronic kidney disease: results from the National Health and Nutrition Examination Survey (NHANES): 2005-2010. BMC Nephrol. 2013; 14(1):178. https://doi.org/10.1186/1471-2369-14-178.

10. Ville AP, Heyman MB, Medrano R, Wojcicki JM. Early antibiotic exposure and risk of childhood obesity in Latinos. Child Obes. 2017;13(3):231-235. https:// doi.org/10.1089/chi.2016.0235. 
11. Wojcicki JM, Prather AA, Epel E, Wang D, Dubal DB. Cord blood klotho levels are inversely associated with leptin in healthy Latino neonates at risk for obesity. J Pediatr Endocrinol Metab. 2018;31(5):515-20. https://doi.org/1 0.1515/jpem-2017-0357.

12. 2000 CDC Growth Charts for the United States. Public Health Service, Centers for Disease Control and Prevention, National Center for Health Statistics; 2002.

13. The World Health Organization's infant feeding recommendation. World Health Organization. https://www.who.int/nutrition/topics/infantfeeding_ recommendation/en/. Published January 29, 2015. Accessed 30 Mar 2021.

14. Prather AA, Epel ES, Arenander J, Broestl L, Garay Bl, Wang D, et al. Longevity factor klotho and chronic psychological stress. Transl Psychiatry. 2015;5(6):e585. https://doi.org/10.1038/tp.2015.81.

15. Chaudry A, Wimer C. Poverty is not just an Indicator: the relationship between income, poverty, and child well-being. Acad Pediatr. 2016;16(3): S23-9. https://doi.org/10.1016/j.acap.2015.12.010.

16. Blair C, Raver CC, Granger D, Mills-Koonce R, Hibel L. The family life project key investigators. Allostasis and allostatic load in the context of poverty in early childhood. Dev Psychopathol. 2011;23(3):845-57. https://doi.org/10.101 7/S0954579411000344.

17. Blair C, Raver CC. Poverty, stress, and brain development: new directions for prevention and intervention. Acad Pediatr. 2016;16(3):S30-6. https://doi. org/10.1016/j.acap.2016.01.010.

18. Cheng C-Y, Kuro-o M, Razzaque MS. Molecular regulation of phosphate metabolism by fibroblast growth Factor-23-Klotho system. Adv Chronic Kidney Dis. 2011;18(2):91-7. https://doi.org/10.1053/.j.ackd.2010.11.007.

19. Kurosu H, Ogawa Y, Miyoshi M, Yamamoto M, Nandi A, Rosenblatt KP, et al. Regulation of fibroblast growth Factor-23 signaling by Klotho. J Biol Chem. 2006;281(10):6120-3. https://doi.org/10.1074/jbc.C500457200.

20. Wickham E. Phosphorus content in commonly consumed beverages. J Ren Nutr. 2014;24(1):e1-4. https://doi.org/10.1053/j.jn.2013.10.002.

21. Bump M. Organic phosphorus versus inorganic phosphorus: empowering adult kidney patients with nutrition education. J Ren Nutr. 2016;26(5):e31-3. https://doi.org/10.1053/j.jn.2016.05.002.

22. Noori N, Sims JJ, Kopple JD, et al. Organic and inorganic dietary phosphorus and its management in chronic kidney disease. Iran J Kidney Dis. 2010;4(2): 89-100.

23. Kutluturk Y, Akinci A, Ozerol IH, Yologlu S. The relationship between serum FGF-23 concentration and insulin resistance, prediabetes and dyslipidemia in obese children and adolescents. J Pediatr Endocrinol Metab. 2019;32(7): 707-14. https://doi.org/10.1515/jpem-2018-0507.

24. Yamamoto M, Clark JD, Pastor JV, Gurnani P, Nandi A, Kurosu H, et al. Regulation of oxidative stress by the anti-aging hormone Klotho. J Biol Chem. 2005;280(45):38029-34. https://doi.org/10.1074/jbc.M509039200.

25. Efthymiadou A, Kritikou D, Mantagos S, Chrysis D. The effect of GH treatment on serum FGF23 and Klotho in GH-deficient children. Eur J Endocrinol. 2016;174(4):473-9. https://doi.org/10.1530/EJE-15-1018.

26. Dubal DB, Yokoyama JS, Zhu L, Broestl L, Worden K, Wang D, et al. Life extension factor Klotho enhances cognition. Cell Rep. 2014;7(4):1065-76. https://doi.org/10.1016/..celrep.2014.03.076.

27. Birch L, Savage JS, Ventura A. Influences on the development of Children's eating Behaviours: from infancy to adolescence. Published online 2009:11.

28. Powell LM, Wada R, Khan T, Emery SL. Food and beverage television advertising exposure and youth consumption, body mass index and adiposity outcomes. Can J Econ Can Décon. 2017;50(2):345-64. https://doi. org/10.1111/caje.12261.

29. Kumar G, Onufrak S, Zytnick D, Kingsley B, Park S. Self-reported advertising exposure to sugar-sweetened beverages among US youth. Public Health Nutr. 2015;18(7):1173-9. https://doi.org/10.1017/S1368980014001785.

\section{Publisher's Note}

Springer Nature remains neutral with regard to jurisdictional claims in published maps and institutional affiliations.

Ready to submit your research? Choose BMC and benefit from:

- fast, convenient online submission

- thorough peer review by experienced researchers in your field

- rapid publication on acceptance

- support for research data, including large and complex data types

- gold Open Access which fosters wider collaboration and increased citations

- maximum visibility for your research: over $100 \mathrm{M}$ website views per year

At BMC, research is always in progress.

Learn more biomedcentral.com/submissions 